\title{
Spinal Cord Tumor Presenting with Abdominal Pain: A Case Report
}

\author{
Anood Alassaf ${ }^{1}$, Rana Al Shami ${ }^{1}$, Jehan Al Rayahi ${ }^{1}$, William Mifsud ${ }^{1}$, Khalid Al Kharazi ${ }^{1}$, \\ and Abdulqadir Nashwan ${ }^{2}$ \\ ${ }^{1}$ Sidra Medical and Research Center \\ ${ }^{2}$ Hamad Medical Corporation
}

May 13, 2021

\begin{abstract}
Three years old boy presented with recurrent severe abdominal pain. He was treated for constipation and the pain resolved. Later he developed a limitation of neck movements. Investigations revealed a spinal cord mass. After surgical resection, histopathology confirmed pilocytic astrocytoma.

Short Title: Spinal Cord Tumor in Three Years Old Child

Spinal Cord Tumor Presenting with Abdominal Pain: A Case Report

Anood Alassaf ${ }^{1,6}$, Rana Al Shami ${ }^{2}$, Jehan Al Rayahi ${ }^{3}$, William Mifsud ${ }^{4}$, Khalid Al-Kharazi ${ }^{5}$, Abdulqadir J. Nashwan ${ }^{6}$

1. Pediatrics Department, Sidra Medical and Research Center, Doha, Qatar

2. Neurology Department, Sidra Medical and Research Center, Doha, Qatar

3. Diagnostic Imaging Department, Sidra Medical and Research Center, Doha, Qatar

4. Anatomical Pathology Unit, Sidra Medical and Research Center, Doha, Qatar

5. Neurosurgery Department, Sidra Medical and Research Center, Doha, Qatar

6. Hamad Medical Corporation, Doha, Qatar

Corresponding author:

Mr. Abdulqadir J. Nashwan

anashwan@hamad.qa
\end{abstract}

Tel: $(+974) 40240487$

Mob: (+974) 66473549

P.O.Box 3050 Doha, Qatar

\section{ABSTRACT}

Three years old boy presented with recurrent severe abdominal pain. He was treated for constipation and the pain resolved. Later he developed a limitation of neck movements. Investigations revealed a spinal cord mass. After surgical resection, histopathology confirmed pilocytic astrocytoma. 
Keywords: Abdominal pain; Astrocytoma; Pilocytic Astrocytoma , Pediatric

\section{Key Clinical Message:}

The authors urge clinicians to observe for early signs of spinal cord tumor in children presenting with unusual presentations such as abdominal pain. Early recognition of spinal cord tumors and immediate treatment will prevent life-threatening complications and improve the prognosis.

\section{BACKGROUND}

Abdominal pain is one of the commonest reasons to seek medical help in pediatrics. It is estimated that about $15 \%$ of children visited doctors because of abdominal pain. Approximately $25 \%$ of these patients had pain that affected their everyday activities. Depending on the characteristics of the abdominal pain, the differential diagnosis includes a variety of gastrointestinal causes like abdominal pain (functional), constipation, and gastroesophageal reflux-related, with other intra-abdominal and extra-abdominal etiologies. Examples of these are oncological processes manifesting with abdominal pain. ${ }^{1,2}$ Herein, we highlight a rare case of pediatric spinal cord tumor presenting as a recurrent abdominal pain.

\section{CASE PRESENTATION}

A three years old boy, previously healthy, presented to the emergency department with severe abdominal pain one month before referral to the pediatric neurology clinic for evaluation of his neck stiffness. The abdominal pain was as periumbilical, occurring in episodes with each episode lasting from few minutes to several hours. It was worse while the child was lying on his back, and It occasionally awakened him from sleep. As the patient was also having hard and infrequent bowel movements, therefore, he was given daily laxative therapy as his symptoms were attributed to constipation. Initial neurologic and abdominal examinations revealed no abnormalities. Magnetic resonance imaging (MRI) of the pelvis and abdomen was performed and reported as normal. Laboratory investigations, including comprehensive metabolic panel, complete blood count, and C-reactive protein (CRP), were also normal. The abdominal pain improved with a high dose of laxative. Both parents reported that the patient repeatedly bent his trunk while walking, with limitation of neck movements. They also noted unexplained night awakening with bouts of hallucination. He was then evaluated in the pediatric neurology clinic at our institution for his new complaints. Clinical examination showed marked limitation of his neck movements in all directions, more so on neck extension. His cranial nerve examination, motor power, tone, and deep tendon reflexes were normal. MRI of the head and neck was done and showed a relatively well-defined thoracic intramedullary cord lesion showing irregular peripheral enhancement and central areas of necrosis (Figure 1). The tumor showed significant perilesional cord edema extending to the cervico-medullary junction superiorly and down to T10 vertebral level. The differential diagnosis included low-grade astrocytoma, ependymoma, and, less likely, ganglioglioma.

The patient underwent C5-T5 laminotomy, and gross total resection of the intradural intramedullary spinal tumor was achieved. Intraoperative neuromonitoring was used.

Histopathology showed a neoplasm of low to moderate cellularity, characterized by a biphasic texture of dense fibrillar areas with bipolar cells and Rosenthal fibers and areas with low fibril density containing cells with multipolar processes and variably mucoid matrix. The nuclei of cells in both areas showed bland features. There was also focal microvascular proliferation; there was no necrosis. Mitotic figures were very sparse. The neoplastic cells were diffusely GFAP positive. Hence, the histological diagnosis was pilocytic astrocytoma (Figure 2).

Post-operative MRI revealed near-complete resection of the spinal tumor with small nodular residual tumor at the cranial extent a well as significant cord decompression with reduced cord edema cranial and caudal to the tumor site (Figure 3).

Clinically the patient was doing well during his follow-up with no neurological deficits.

\section{DISCUSSION}


Approximately $10 \%$ of all pediatric central nervous neoplasms are located in the spinal cord, either intramedullary or extramedullary. ${ }^{3}$ It is estimated that $90 \%$ of intramedullary spinal cord masses are glial tumors. ${ }^{4}$ These mainly include astrocytoma and ependymoma. ${ }^{5}$ All age groups be can be affected by these neoplasms; however, they occur more frequently in children less than 10 years of age. Overall, males and females are equally affected. ${ }^{5}$ The growth of the spinal cord tumors are slow, and symptoms are usually nonspecific, which is the reason why these tumors are frequently diagnosed at later stages. ${ }^{5}$ Patients typically have symptoms that tend to evolve over months to years. Most commonly, regional back pain is the complaint at first presentation. ${ }^{6}$

Fortunately, low-grade tumors comprise most spinal cord tumors. However, high-grade lesions account for only ten to fifteen percent of pediatric tumors, and they tend to occur in a slightly higher proportion in adults. ${ }^{6}$

Astrocytomas are slow-growing tumors. Typically, the interval between the onset of symptoms and the time of diagnosis is long and might take months or even years. ${ }^{6}$ Two thirds of the patients reported diffuse back pain as the initial manifestation. The pain they described was worse with lying down, causing nighttime symptoms. ${ }^{1,5}$

In France between 1971 and 1994, a review of 73 cases of spinal cord astrocytoma in pediatrics revealed that back pain was the most common presentation (89\%), while $78 \%$ noticed to have gait abnormalities and $32 \%$ developed weakness in the upper limbs or sphincter disturbances. Fewer than $10 \%$ of the symptoms were headache and muscle weakness. ${ }^{3}$

Our patient did not initially present with back pain but rather with abdominal pain and with leaning forward while walking.

Regarding the modality of choice for diagnosis, MRI has excellent soft-tissue contrast. At the same time, it lacks ionizing radiation making it safe for children. There is still a debate about the treatment of many of these neoplasms. Still, the best option indicated by the current evidence is microsurgical resection, with adjunctive treatment being reserved in cases of recurrent or high-grade lesions. ${ }^{7}$

\section{CONCLUSION}

While most causes of abdominal pain are benign and can be diagnosed with minimal investigations, other causes warrant more advanced diagnostic. Especially where there is a significant recurrent abdominal pain, which can be worse at night with no identifiable gastrointestinal etiology, extra-intestinal causes, including spinal cord tumor, should be considered.

\section{Abbreviations}

MRI: Magnetic resonance imaging

CRP: C-reactive protein

\section{Declarations}

\section{Ethics approval and consent to participate}

The article describes a case report. Therefore, no additional permission from our Ethics Committee was required.

\section{Consent for publication}

The consent for publication was obtained.

\section{Availability of data and material}

All data generated or analyzed during this study are included in this published article.

\section{Competing interests}


The authors declare that they have no competing interests.

\section{Funding}

This study was not funded.

\section{Authors' contributions}

AAF, RSH, JAR, WIM, KAK, AJN: Data Collection, Literature Search, Manuscript Preparation

All authors read and approved the final manuscript

\section{Acknowledgments}

Open Access funding provided by the Qatar National Library.

\section{REFERENCES}

1. Jackson K, Lapsia S, Strunc M, Tye G. Spinal cord astrocytoma: a unique presentation of abdominal pain. Radiology case reports.2018;13(1):284-288.

2. Kothbauer KF. Neurosurgical management of intramedullary spinal cord tumors in children. Pediatric neurosurgery. 2007;43(3):222-235.

3. Menashe SJ, Iyer RS. Pediatric spinal neoplasia: A practical imaging overview of intramedullary, intradural, and osseous tumors. Current problems in diagnostic radiology. 2013;42(6):249-265.

4. Arnautović KI, Gokaslan ZL. Spinal Cord Tumors. Springer; 2019.

5. Smith AB, Soderlund KA, Rushing EJ, Smirniotopolous JG. Radiologic-pathologic correlation of pediatric and adolescent spinal neoplasms: Part 1, Intramedullary spinal neoplasms. American Journal of Roentgenology. 2012;198(1):34-43.

6. Houten JK, Cooper PR. Spinal cord astrocytomas: presentation, management and outcome. Journal of neuro-oncology.2000;47(3):219-224.

7. Bouffet E, Pierre-Kahn A, Marchal JC, et al. Prognostic factors in pediatric spinal cord astrocytoma. Cancer: Interdisciplinary International Journal of the American Cancer Society.1998;83(11):2391-2399.

\section{FIGURES LEGENDS}

Figure 1: Preoperative MRI of the spine. Sagittal T2 (a), T1 (b) and gadolinium enhanced T1 weighted images (c) demonstrate a relatively well-defined intramedullary T2 hyperintense, T1 hypointense expansile cord lesion spanning C5 down to T5 vertebral levels with Significant perilesional cord edema extending to the cervicomedullary junction superiorly and down to T10 vertebral level. The tumors showing irregular, mainly peripheral enhancement and central areas of necrosis.

Figure 2: Microphotographs showing the histological features of the lesion. A: A mostly dense textured area with Rosenthal fibres and bipolar fibrillary cells. B: An area with loose texture. C: Focal microvascular proliferation. D: Ki67 immunohistochemistry showing a very low mitotic proportion of neoplastic cells. Scale bars: $200 \mu \mathrm{m}$.

Figure 3: Post-operative MRI the spine. Sagittal T2 (a) and gadolinium enhanced T1 weighted images (b) show near complete resection of the spinal tumor with small nodular residual tumor at the cranial extent a well as significant cord decompression with reduced cord edema cranial and caudal to the operative site.

\section{Hosted file}

Fig1.pdf available at https://authorea.com/users/413761/articles/521960-spinal-cord-tumorpresenting-with-abdominal-pain-a-case-report

\section{Hosted file}


Fig2.pdf available at https://authorea.com/users/413761/articles/521960-spinal-cord-tumorpresenting-with-abdominal-pain-a-case-report

\section{Hosted file}

Fig3.pdf available at https://authorea.com/users/413761/articles/521960-spinal-cord-tumorpresenting-with-abdominal-pain-a-case-report 